\title{
Aplicación de la regresión cuantílica para predecir el volumen fustal: Estudio de caso
}

\section{Application of quantile regression to predict stem volume: Case study}

\author{
Adan Nava-Nava ${ }^{1}$, Pablo Antúnez ${ }^{2 *}$ \\ ${ }^{1}$ Maestría en Ciencias en Conservación de los Recursos Forestales. Universidad de la Sierra Juárez. Avenida Universidad s/n, \\ Ixtlán de Juárez, CP. 68725. Oaxaca, México \\ ${ }^{2}$ División de Estudios de Posgrado-Instituto de Estudios Ambientales. Universidad de la Sierra Juárez. Avenida Universidad \\ s/n, Ixtlán de Juárez, CP. 68725. Oaxaca, México \\ *Autor de correspondencia: pantunez4@gmail.com
}

Nota científica recibido: 09 de junio de 2017 aceptado: 21 de abril de 2018

RESUMEN. En la estimación del volumen fustal de los árboles, es deseable contar con herramientas matemáticas precisas en las que se incorpore la menor cantidad de insumos para un control adecuado de la extracción de madera en rollo de un bosque bajo aprovechamiento. El objetivo fue examinar si la regresión cuantílica puede predecir con mayor precisión el volumen fustal de Pinus patula Schiede ex Schltdl. \& Cham que las técnicas convencionales, usando el diámetro normal como predictor. Se usaron datos de 148 árboles dominantes y codominantes para generar tres rectas de regresión cuantílica. El ajuste y aplicación de la regresión propuesta se comparó con la obtenida con el modelo exponencial de Berkhout con ajuste por mínimos cuadrados no lineales. Los resultados sugieren que esta técnica mejora la predicción volumétrica de los árboles al generar rectas en distintos segmentos de la distribución condicional, permitiendo una reducción significativa del error de predicción para los datos alejados de la media aritmética.

Palabras clave: Ecuaciones de volumen, datos heterogéneos, reducción de insumos, homocedasticidad de residuos

ABSTRACT. In estimating the stem volume of trees, it is desirable to have precise mathematical tools in which the least amount of inputs is incorporated to achieve adequate control over the extraction of roundwood from a forest under exploitation. The objective was to determine whether quantile regression can more accurately predict the stem volume of Pinus patula Schiede ex Schltdl. \& Cham than conventional techniques, using diameter at breast height as a predictor. Data from 148 dominant and co-dominant trees were used to generate three quantile regression lines. The fitting and application of the proposed regression were compared with that obtained with the Berkhout exponential model with fitting by non-linear least squares. The results suggest that this technique improves the volumetric prediction of the trees by generating lines in different segments of the conditional distribution, allowing a significant reduction in the prediction error for the data far from the arithmetic mean.

Key words: Volume equations, heterogeneous data, input reduction, homoscedasticity of residuals

\section{INTRODUCCIÓN}

Una de las variables claves para la acertada toma de decisiones en el manejo de los recursos maderables, es el volumen de los árboles en pie (Clutter et al. 1983). De forma convencional, el volumen de cada árbol se estima a partir del diámetro normal y la altura total, para luego extrapolar la información a todo el rodal o bosque bajo manejo (Carrillo et al. 2004). Para la es- timación del volumen fustal, se debe contar con herramientas matemáticas precisas, que incorporen la menor cantidad posible de variables de bajo costo, lo que permite un estricto control de la extracción de madera en rollo, base para una mejor planificación del aprovechamiento sustentable de los bosques (Romahn y Ramírez-Maldonado 2010, MartínezLópez y Acosta-Ramos 2014). Pero muchas veces se tiene una base de datos con individuos de diferentes edades y tamaños, lo que propicia una 
alta dispersión de la información, lo que causa que los valores más alejados de la media aritmética no sean explicados con propiedad por las técnicas de regresión convencionales, en particular, con el método de Mínimos Cuadrados Ordinarios (MCO) o en su forma no lineal (MCNL) que generan una línea y curva de regresión, según sea el caso, basada en el promedio (Galton 1890).

El uso de Mínimos Cuadrados (MC), tanto en su forma lineal como en su forma no lineal, implica asumir propiedades paramétricas como la distribución normal de los datos, la homogeneidad de la varianza, la independencia de los regresores y la ausencia de colinealidad (Dismuke y Lindrooth 2006). El incumplimiento de algunos de estos supuestos, limita su aplicación; por ejemplo, si se detecta colinealidad, no deben usarse los MCO, debido a que se contraviene al soporte teórico-matemático del método (Weeraratne 2016). Muchas veces, se deben transformar los datos originales sacándole raíz cuadrada, logaritmo natural, incluir variables de ponderación en los residuales o hacer cálculos adicionales para intentar solventar estas restricciones (Box y Cox 1964, Cailliez 1980, Zimmerman 1994). Una alternativa en silvicultura y ecología forestal, es la regresión de cuantiles $(\mathrm{RC})$, que permite generar rectas de regresión a cualquier porción dada de una variable respuesta, definida por su distribución de probabilidad (Koenker y Bassett 1978, Cade y Noon 2003). Una de las ventajas de la $R C$ radica en su flexibilidad para evaluar el efecto de una variable aleatoria sobre la distribución de una variable de interés, al generar una familia de modelos de regresión, uno para cada cuantil de la distribución condicional (Sosa-Escudero 2005), mientras que la regresión ordinaria sólo estima los efectos medios condicionales de las variables independientes (Koenker y Bassett 1978, Sosa-Escudero 2005).

La regresión cuantílica se usa principalmente en las ciencias sociales, y muy poco en las ciencias biológicas. En las ciencias sociales, se ha utilizado para estimar la elasticidad del precio en la demanda de alcohol (Manning et al. 1995); el efecto de la educación de los padres y su edad sobre la educación de los hijos (Di Gresia et al. 2005); y en el área forestal se ha propuesto para estudiar la producción de bellotas en un bosque (Cade et al. 1999), para predecir el crecimiento de los árboles (Bohora y Cao 2014) o para determinar la relación alométrica entre el diámetro y la copa de los árboles (Pretzsch et al. 2015). Por lo anterior, el objetivo del trabajo fue evaluar la efectividad de la regresión cuantílica para modelar el volumen fustal de Pinus patula Schl. et Cham. en función del diámetro normal comparando sus resultados con los de un modelo tradicional.

\section{MATERIALES Y MÉTODOS}

\section{Área de estudio}

El estudio se realizó en los bosques templados de Santiago Comaltepec, en la Sierra Norte del estado de Oaxaca, al suroeste de México, entre las coordenadas $17^{\circ} 43^{\prime} 36^{\prime \prime}$ - $17^{\circ} 32^{\prime} 50^{\prime \prime}$ LN y $96^{\circ} 35^{\prime} 12^{\prime \prime}$ - $96^{\circ} 16^{\prime} 02^{\prime \prime}$ LO. El predio tiene una superficie de $26.5 \mathrm{~km}^{2}$ (Figura 1), la elevación sobre el nivel del mar fluctúa entre los 1800 y $3000 \mathrm{~m}$. La temperatura media máxima anual es de $13.4{ }^{\circ} \mathrm{C}$, con mínima anual de $4.7{ }^{\circ} \mathrm{C}$. La precipitación en el verano oscila entre los 600 y $1200 \mathrm{~mm}$, y en la temporada de secas la precipitación media es de $225.5 \mathrm{~mm}$ con temperatura media de $16{ }^{\circ} \mathrm{C}$ (INEGI 2002, INEGI 2018).

\section{Muestreo y variables}

Los datos usados provienen de una muestra de 148 árboles dominantes y codominantes de $\mathrm{Pi}$ nus patula registrados en 60 parcelas temporales de $1000 \mathrm{~m}^{2}$ distribuidas sistemáticamente en el área de estudio, tamaño de parcela común en el inventario forestal con fines de aprovechamiento forestal. Para predecir el volumen fustal con corteza, se usó como predictor el diámetro normal, a $1.30 \mathrm{~m}$ del nivel del suelo, al ser una de las variables indicadoras confiables de las dimensiones de los árboles, que se usa para predecir el volumen, para definir la estructura de un rodal o clasificar una masa forestal, entre otros usos (Gadow et al. 2007, Návar 2009). El diámetro normal se obtuvo con una forcípula y el volumen observado se generó con la ecuación tradicional de Schumacher y Hall (1933) al integrar el 


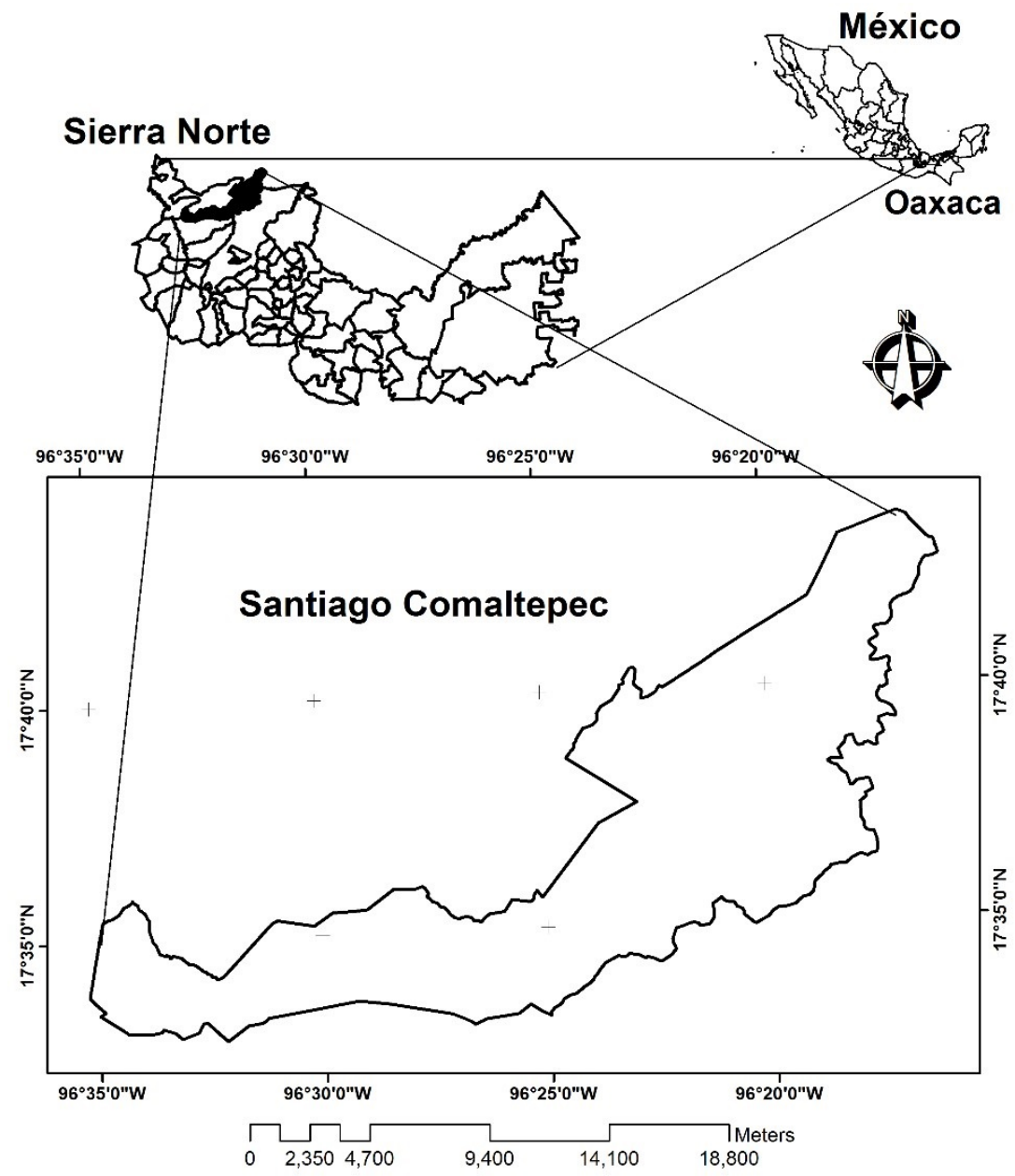

Figura 1. Localización del área de estudio.

diámetro normal y la altura total de los 148 individuos que conformaron la muestra. La estadística descriptiva de las variables usadas se muestra en la Tabla 1.

Tabla 1. Resumen estadístico de la muestra.

\begin{tabular}{cccccc}
\hline Variable & Media & $\mathrm{CV}$ & $\mathrm{CA}$ & Máximo & Mínimo \\
\hline $\mathrm{Dn}$ & 53.5 & 34.6 & 1.88 & 140 & 30 \\
$\mathrm{H}$ & 28.2 & 27.5 & 0.23 & 46 & 12 \\
$\mathrm{~V}$ & 3.3 & 99.7 & 2.79 & 20.41 & 0.58 \\
\hline Dn: diámetro normal $(\mathrm{cm}), \mathrm{H}$ : altura total $(\mathrm{m}), \mathrm{V}:$ \\
volumen $\left(\mathrm{m}^{3}\right), \mathrm{CV}$ : coeficiente de variación $(\%), \quad \mathrm{CA}:$ \\
coeficiente de asimetría.
\end{tabular}

\section{Análisis de datos}

Para probar si la regresión de cuantiles puede mejorar la predicción del volumen fustal de la es- pecie estudiada, se usó solo el diámetro normal como predictor, usando de referencia el modelo exponencial de Berkhout (Loetsch et al. 1973) por ser uno de los mejores modelos de una entrada que predice el volumen fustal de especies de pino con errores mínimos (Bonyad y Rostami 2005, Wardasanti 2011, Ramírez-Martínez et al. 2016), cuya expresión es: $V=\beta_{0} \cdot D N^{\beta_{1}}$ Donde: $\mathrm{V}$ : volumen $\left(\mathrm{m}^{3}\right)$, DN: diámetro normal $(\mathrm{cm}), \beta_{i}$ : parámetros de regresión.

Con el método de la regresión cuantílica (Koenker y Bassett 1978, Koenker y Mizera 2004), se generaron las rectas de regresión de los cuantiles de $0.25,0.50$ y 0.75 , procurando abarcar la variabilidad de los datos por encima y por debajo de 
la curva de regresión generada con MCNL. La definición analítica de la regresión cuantílica es: Sea $Y$ una variable aleatoria cualquiera, definida por una función $F_{Y}($.$) , de la forma: F_{Y}(y)=\operatorname{prob}(Y \leq$ $y)=\tau$

Tal que, para cualquier valor $0<\tau<1, Q(\tau)$ $=\inf y: F(y) \geq \tau$ corresponde al $\tau$-ésimo quantil de $X$. En este sentido, la mediana $Q(1 / 2)$, juega un papel esencial; $y$, del mismo modo que la función de distribución, la función cuantílica proporciona una caracterización de la variable aleatoria de interés, $Y$. De esta forma, un cuantil puede ser planteada como la solución a un problema de optimización simple. Tal que, para cualquier valor $0<\tau<1$, puede definirse como:

$$
\rho \tau(u)=u(\tau-I(u<0))
$$

Donde: $I($.$) denota la función indicadora. Al$ minimizar la esperanza de $\rho \tau(Y-\xi)$, con respecto a $\xi, \xi(\tau)$, la más pequeña de las cuales está en $Q(\tau)$. Analógicamente, la muestra de $Q(\tau)$, que proviene de una muestra aleatoria $y 1, \ldots, y n$, de $Y^{\prime} s$, correspondería a la $\tau$-ésima muestra cuantílica, de modo que el problema de optimización se puede expresar como:

$$
\min _{\xi \in R} \sum_{i=1}^{n} \rho \tau(y i-\xi)
$$

Los planteamientos teóricos de la regresión de cuantiles, la ilustración gráfica de su función de verificación, y otras ecuaciones derivadas se encuentran en Koenker y Bassett (1978) y Koenker y Mizera (2004). La contribución de las constantes de cada recta cuantílica se valoró usando un nivel de significancia de $\alpha=0.05$ (Di Rienzo et al. 2005) haciendo una corrección de Bonferroni con el fin de reducir en lo posible la probabilidad de cometer el error tipo I (Hochberg 1988). Al no observarse una alta variabilidad de los datos (Tabla 1), se generaron solo tres rectas cuantílicas, en los cuantiles $0.25,0.50$ y 0.75 , las cuales cubrieron la relativa dispersión de los datos.

Para evaluar el ajuste de las rectas ajustadas de cada cuantil, se usaron los criterios de ajuste de análisis gráfico de los residuales y el criterio de información de Akaike (AIC), que mide la calidad relativa del ajuste de un modelo, al medir la diferencia entre la proyección del modelo y la realidad basada en el criterio teórico de la información mínima en el subconjunto $Q(\tau)$ asociado a un $\tau$-ésimo cuantil, cuando menor es el valor de AIC, más admisible es el modelo (Akaike 1974). En un inicio se obtuvo un pseudo valor de ajuste $\left(R^{1}\right)$ para cada recta cuantílica, al comparar la suma de desviaciones ponderadas para el modelo completo con la misma suma del modelo (Koenker y Machado 1999); al no poder compararse con los indicadores de ajuste del modelo de Berkhout el cuál se ajustó por MCNL, estos coeficientes no se incluyeron; pero se estimaron coeficientes de determinación ajustado $\left(\mathrm{R}^{2}{ }_{a d j}\right)$ y el error residual para cada cuantil para contrastar con mayor claridad el rendimiento de cada modelo. Los valores de $\mathrm{R}_{a d j}^{2}$, se obtuvieron al extraer y reajustar los valores del subconjunto $Q(\tau)$. En este caso, $\mathbf{R}_{a d j}^{2}$, representa el porcentaje de variación de los resultados, explicado por una recta cuantílica, asumiendo que cada recta se asocia a un intervalo definido de valores de la $\tau$-ésima regresión cuantílica. Para el ajuste de los modelos se usó el paquete quantreg de R (Koenker et al. 2015). Los modelos se evaluaron mediante una validación cruzada con una muestra independiente de 50 individuos. Finalmente, los resultados se ejemplificaron buscando la ecuación cuantílica que redujera el error de predicción según la categoría diamétrica de los árboles.

\section{RESULTADOS Y DISCUSIÓN}

Los coeficientes paramétricos con sus respectivos valores de $p$-value de las diferentes rectas cuantílicas y del modelo no lineal de Berkhout se muestran en la Tabla 2. Con base en los indicadores de ajuste, el modelo no lineal de Berkhout ajustado por MCNL fue superado en la mayoría de los casos. Tomando en cuenta el $\mathrm{R}^{2}$ adj los valores en los cuantiles de $0.25,0.50$ y 0.75 fueron de $0.94,0.99$ y 0.99 , respectivamente; mientras que para el modelo de Berkhout fue de 0.94 (Tabla 2). Al comparar 
Tabla 2. Parámetros e indicadores de ajuste para cada recta cuantílica y para el modelo de Berkhout ajustado por mínimos cuadrados no lineales.

\begin{tabular}{|c|c|c|c|c|c|c|}
\hline \multicolumn{7}{|c|}{ Mínimos cuadrados no lineales } \\
\hline Modelo & & Estimación & $P>|t|$ & $\mathrm{AIC}$ & $\mathrm{R}_{a d j}^{2}$ & Error residual \\
\hline Berkhout & $\begin{array}{l}\beta_{0} \\
\beta_{1}\end{array}$ & $\begin{array}{l}0.000591 \\
2.138211\end{array}$ & $\begin{array}{l}<0.0001^{*} \\
<0.0001^{*}\end{array}$ & 237.1086 & 0.94 & 0.795 \\
\hline \multicolumn{7}{|c|}{ Regresión cuantílica } \\
\hline $\mathrm{t}=0.25$ & $\begin{array}{l}\beta_{0} \\
\beta_{1}\end{array}$ & $\begin{array}{c}-4.17824 \\
0.12457\end{array}$ & $\begin{array}{l}<0.0001^{*} \\
<0.0001^{*}\end{array}$ & 33.4014 & 0.94 & 0.353 \\
\hline$t=0.50$ & $\begin{array}{l}\beta_{0} \\
\beta_{1}\end{array}$ & $\begin{array}{c}-5.011244 \\
0.155982\end{array}$ & $\begin{array}{l}<0.0001^{*} \\
<0.0001^{*}\end{array}$ & -31.7449 & 0.99 & 0.125 \\
\hline $\mathrm{t}=0.75$ & $\begin{array}{l}\beta_{0} \\
\beta_{1} \\
\end{array}$ & $\begin{array}{c}-5.669588 \\
0.185926 \\
\end{array}$ & $\begin{array}{l}<0.0001^{*} \\
<0.0001^{*}\end{array}$ & 47.7839 & 0.99 & 0.480 \\
\hline
\end{tabular}

AIC: Criterio de información Akaike; $\mathrm{R}^{2}{ }_{a d j}$ : Coeficiente de determinación ajustado, $\beta_{0}$ y $\beta_{1}$ : Parámetros estimados para cada modelo." "*" Parámetros significativos con un nivel de significancia de 0.01

los errores de los modelos, se observó un error de predicción de $0.353 \mathrm{~m}^{3}$ en el cuantil $0.250,0.125$ $\mathrm{m}^{3}$ en el cuantil 0.50 y $0.408 \mathrm{~m}^{3}$ en el 0.75 ; en contraste, con el método de mínimos cuadrados no lineales se observó un error de $0.795 \mathrm{~m}^{3}$. De igual forma al contrastar los valores de AIC, con la técnica de MCNL se obtuvo un valor mayor en comparación con los obtenidos con RC. En todos los casos, las constantes fueron significativas $(p \leq 0.01)$ al hacer la corrección de Bonferroni.

Con las dos técnicas, se observa que la línea proyectada por MCNL solo divide en dos porciones la nube de puntos, mientras que la regresión cuantílica intenta cubrir en lo posible la dispersión de los datos al trazar tres rectas (Figura 2a y 2b). Otras de las ventajas identificadas del RC sobre MCNL, es que la primera técnica puede modelar con mayor robustez una categoría diamétrica específica. En un análisis complementario se comprobó que usando la ecuación del cuantil 0.25 , el error de predicción disminuye de forma significativa para los árboles con diámetros entre 55 y $70 \mathrm{~cm}$.

Los datos estudiados mostraron baja dispersión, de modelarse variables con alta variabilidad, los datos extremos quedarían alejados de la curva trazada por el modelo de Berkhout, ocasionando una subestimación o sobreestimación del volumen fustal (Figura 2a y 2b), error que no se refleja en los indicadores de ajuste, ya que la línea de regresión por MCNL se traza en función de la media (Galton 1890). Una forma de solucionar la alta heterogenei- dad de los datos, es trazar tantas rectas como sean necesarias a fin de cubrir los datos extremos (Cade et al. 1999, Cade y Noon 2003; Koenker y Mizera 2004).

Al contrastar los residuos de los dos métodos en un mismo gráfico, se observó un mayor error con los datos del modelo tradicional, lo cual es causado por los valores extremos, los cuales se redujeron usando la ecuación del cuantil 0.75 (Figura 3). El método de MCNL comete un error significativo en las secciones donde la distribución de la variable condicional no es, el cual es corregido parcialmente por la regresión cuantílica al usar las ecuaciones de los cuantiles 0.25 y 0.50 , al mejorar la predicción (Figura 3). Con el modelo de Berkhout, se observó una alta heterocedasticidad en los residuales; en contraste, las rectas cuantílicas corrigen este problema. De igual forma, los residuos estudentizados de las distintas rectas cuantílicas se distribuyen dentro de la franja del intervalo de confianza al 95\%, como el caso del cuantil 0.50 (Figura 4a), mientras que los residuos de los valores extremos del modelo de Berkhout quedaron fuera de este intervalo (Figura 4b).

La correcta aplicación de la regresión cuantílica radica en la adecuada identificación de la fracción de datos susceptibles a ser predichos por la línea o curva cuantílica con el menor error. Usando los parámetros estimados, los modelos finales son: (1) $V=0.000591^{*} D N^{2.138211}$, que corresponde al modelo ajustado por el método de mínimos cuadra- 
A

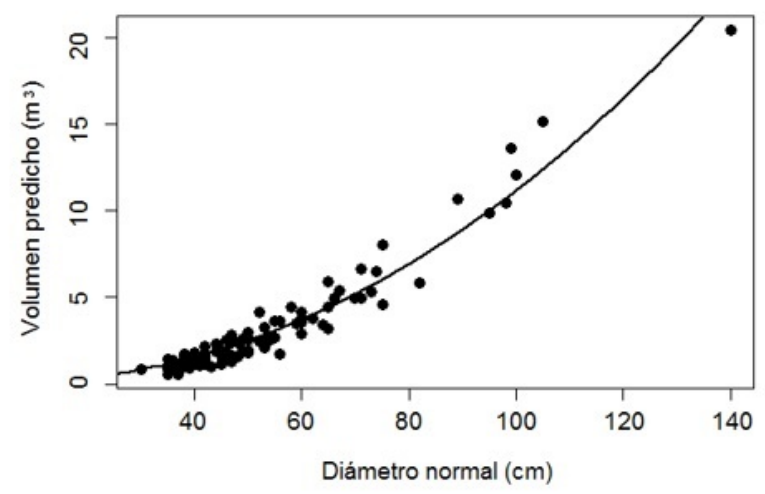

B

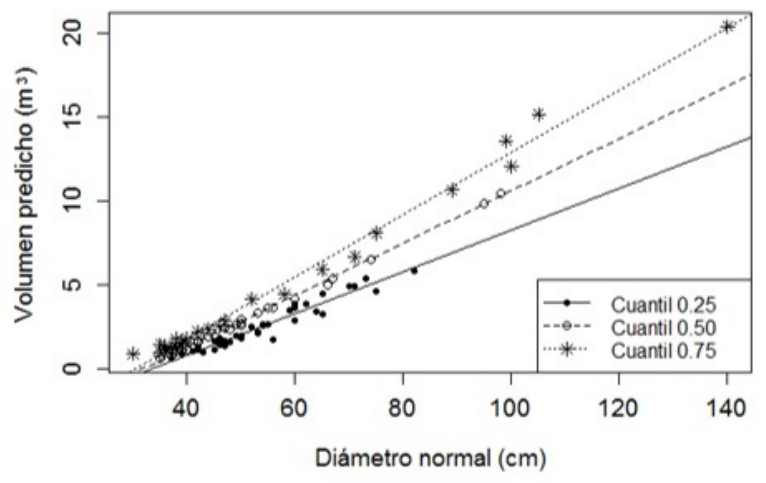

Figura 2. Comportamiento grafico del ajuste del modelo de Berkhout por mínimos cuadrados no lineales (a) y regresión de cuantiles (b).

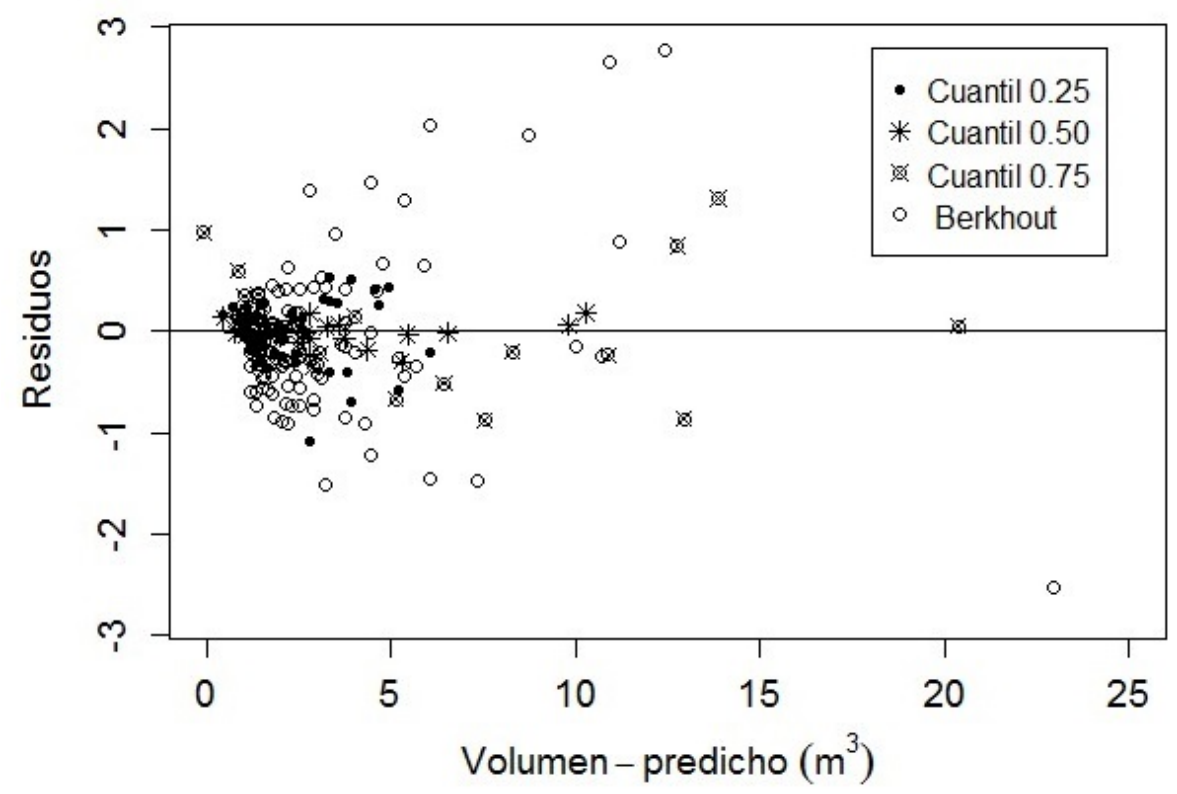

Figura 3. Comportamiento grafico de predichos frente a residuales con los modelos ajustados.

dos; (2) $V=-4.17824+0.12457^{*} D N$ correspondiente al $Q(\tau=0.25)$ ajustado con $\mathrm{QR}$, que fue apropiado para predecir el volumen de árboles con categorías diamétricas de 55 a $80 \mathrm{~cm}$, excluyendo la categoría de $75 \mathrm{~cm}$; (3) $V=-5.011244+$ $0.155982^{*} D N$, modelo final del $Q(\tau=0.50)$, un modelo adecuado para modelar el volumen de árboles con las categorías diamétricas de 40-50, 75 y
$95 \mathrm{~cm}$; y (4) $V=-5.669588+0.185926^{*} D N$, correspondiente al $Q(\tau=0.75)$, con óptimo rendimiento para árboles con categorías diamétricas de $30-35 \mathrm{~cm}$, de 90 y de 100 a $140 \mathrm{~cm}$.

Con estos modelos, se presentan los resultados de los dos métodos con medidas de un árbol proveniente de una muestra independiente, para realizar una validación cruzada, con un diámetro de 
A

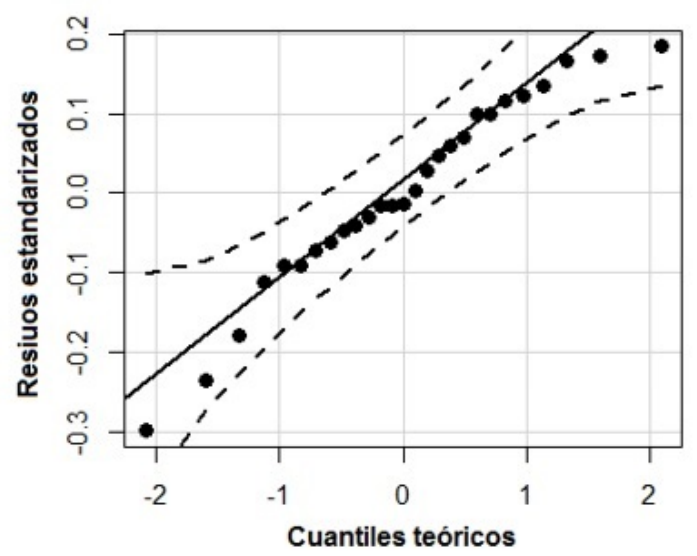

B

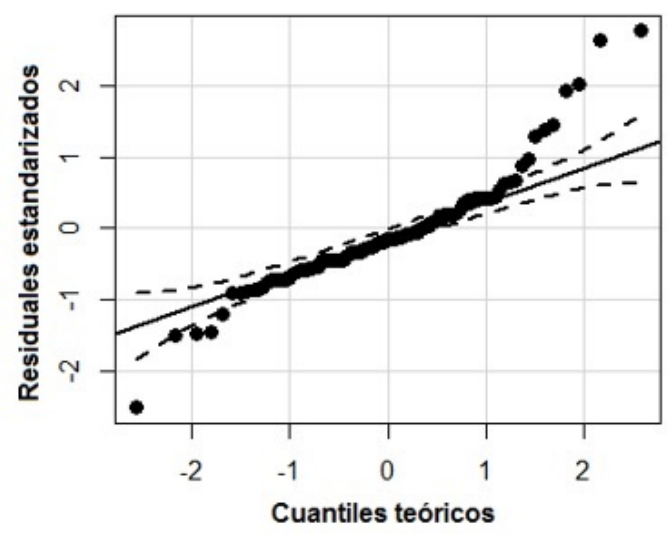

Figura 4. Residuales para la recta cuantílica de $\tau=0.50$ (a) y residuales del modelo de Berkhout (b) con una confiabilidad de $95 \%$.

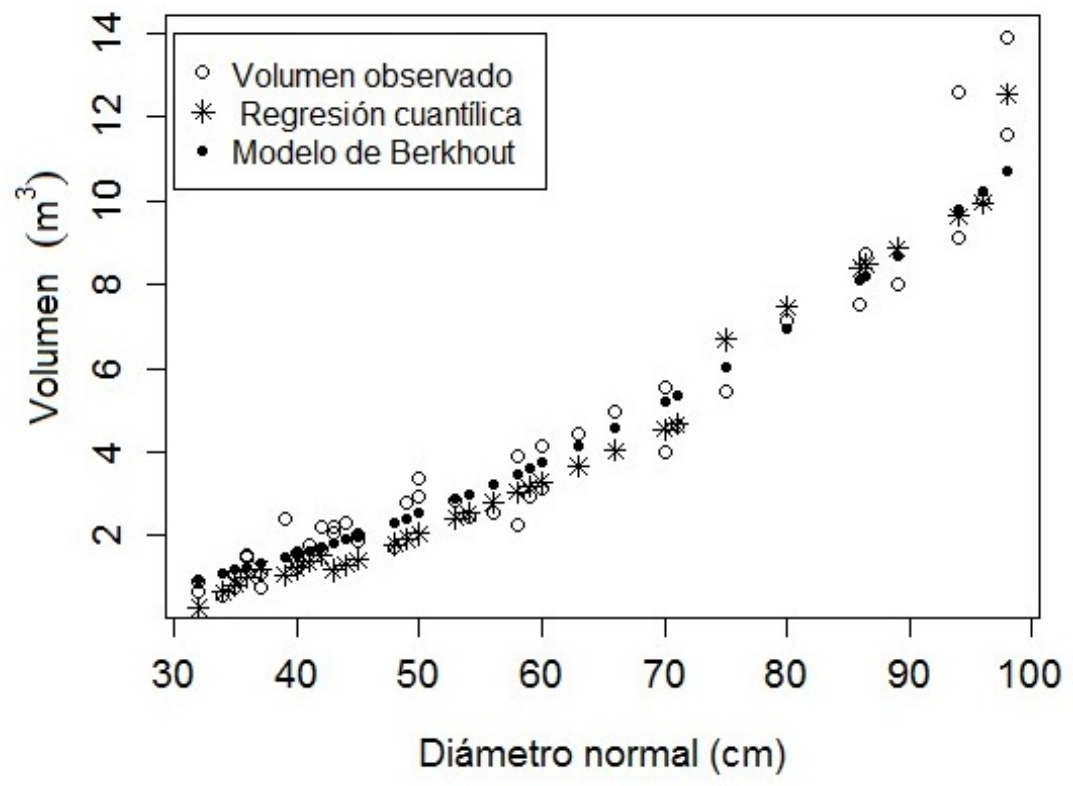

Figura 5. Comportamiento grafico del volumen fustal estimado con los modelos evaluados al realizar la validación cruzada.

$35 \mathrm{~cm}$ y volumen observado de $0.8420 \mathrm{~m}^{3}$. Con el método de MCNL se proyecta un volumen de 1.1834 $\mathrm{m}^{3}$, mientras que el método RC proyecta un volumen de $0.8378 \mathrm{~m}^{3}$; con la ecuación de $Q(\tau=0.75)$ que es el modelo apropiado para esta categoría diamétrica, observándose que el modelo de MCNL comete un error de $0.341 \mathrm{~m}^{3}$ en tanto que con $\mathrm{RC}$ es de $0.004 \mathrm{~m}^{3}$. Aparentemente es un error pequeño; pero al multiplicar por el número de individuos de un rodal o predio, se incremente el error. Debe tomarse en cuenta que en este estudio se generaron rectas, pero la predicción puede mejorarse si se generan 
líneas suavizadas en cada cuantil ajustadas mediante una regresión local.

Al contrastar los modelos ajustados con una muestra independiente, se validó que cada recta cuantílica cubre la dispersión de los datos extremos (Figura 5); en cambio, el modelo de Berkhout, a pesar de ser un modelo de tipo exponencial, excluye los valores extremos. Se concluye que al usar de forma apropiada la regresión cuantílica, la predicción del volumen fustal de las especies maderables como el Pinus patula, puede predecirse con mayor precisión, usando algún atributo de fácil medición, como el diámetro normal, lo que la convierte en una herramienta potencial para modelar datos del área forestal.

\section{LITERATURA CITADA}

Akaike $\mathrm{H}$ (1974) A new look at the statistical model identification. IEEE transactions on automatic control 19: $716-723$.

Cailliez F (1980) Estimación del volumen forestal y predicción del rendimiento, con referencia especial a los trópicos (No. CIDAB-SD391-F6e-22/2). Organización de las Naciones Unidas para la Agricultura y la Alimentación.http://www.fao.org/docrep/016/ap353s/ap353s00.pdf. Fecha de consulta: 20 de abril de 2018.

Bohora SB, Cao QV (2014) Prediction of tree diameter growth using quantile regression and mixed-effects models. Forest Ecology and Management 319: 62-66.

Bonyad SA, Rostami T (2005) Study on Slash pine (Pinus elliottii) as a short rotation forestry in the North of Iran. Caspian Journal of Environmental Sciences 3: 55-58.

Box GE, Cox DR (1964) An analysis of transformations. Journal of the Royal Statistical Society. Series B (Methodological) 26: 211-252

Cade B, Noon B (2003) A gentle introduction to quantile regression for ecologists. Frontiers in Ecology and the Environment 1: 412-420.

Cade B, Terrell J, Schroeder R (1999) Estimating effects of limiting factors with regression quantiles. Ecology 80: $311-323$

Carrillo AF, Acosta MM, Tenorio GG, Becerra FL (2004) Tabla de volumen para Pinus patula Schl. et Cham. en el estado de Hidalgo. Folleto Técnico Núm. 2. Instituto Nacional de Investigaciones Forestales, Agrícolas y Pecuarias. México. 21p.

Clutter JL, Forston JC, Pienaar LV, Brister GH, Bailey RL (1983) Timber management: a quantitative approach. John Wiley \& Sons, Inc. New York, USA. 333p.

Di Gresia L, Fazio M, Porto A, Ripani L, Sosa-Escudero W (2005) Rendimiento y productividad de los estudiantes. El caso de las Universidades Públicas Argentinas. En: Porto A (ed.) Economía de la educación universitaria: Argentina-Brasil-Perú. Editorial de la Universidad Nacional de La Plata, La Plata, Argentina. pp: 5-28.

Di Rienzo DJA, Casanoves F, Gonzalez LA, Tablada EM, Díaz MP, Robledo CW, et al. (2005) Estadística para las ciencias agropecuarias. 6ta ed. Córdoba, Argentina. 378p.

Dismuke C, Lindrooth R (2006) Ordinary least squares. Methods and Designs for Outcomes Research 93: 93-104.

Gadow KV, Sánchez OS, Álvarez JG (2007) Estructura y crecimiento del bosque. Universidad de Göetingen. IUFRO World Series Vol. 12. Alemania. 242p. 
Galton F (1890) Kinship and correlation. The North American Review 150: 419-431.

Hochberg Y (1988) A sharper Bonferroni procedure for multiple tests of significance. Biometrika 75: 800-802.

INEGI (2002) Instituto Nacional de Estadística y Geografía. Anuario estadístico del estado de Oaxaca 20001. INEGI- Gobierno Constitucional del estado de Oaxaca. Tomo I y II. México. 956p.

INEGI (2018) Instituto Nacional de Estadística y Geografía. Prontuario de información geográfica municipal de los Estados Unidos Mexicanos. http://www3.inegi.org.mx/contenidos/app/mexicocifras/datos_geograficos/20/20458.pdf. Fecha de consulta: 05 de abril de 2018.

Koenker R, Bassett G (1978) Regression quantiles. Econometrica 46: 33-50.

Koenker R, Machado J (1999) Goodness of fit and related inference processes for quantile regression. Journal of the American Statistical Association 94: 1296-1310.

Koenker R, Mizera I (2004) Penalized triograms: Total variation regularization for bivariate smoothing. Journal of the Royal Statistical Society: Series B (Statistical Methodology) 66: 145-163.

Koenker R, Portnoy S, Ng PT, Zeileis A, Grosjean P, Ripley BD (2015) Quantile Regression; Package 'quantreg'. http://cran.r-project.org/web/packages/quantreg/quantreg.pdf. Fecha de consulta: 15 de noviembre de 2016.

Loetsch F, Zohrer F, Haller KE (1973) Forest inventory 2. BLV. Verlagsgesellschaft. München, Germany, $469 p$.

Manning W, Blumberg L, Moulton L (1995) The demand for alcohol: the differential response to price. Journal of Health Economics 14: 123-148.

Martínez-López J, Acosta-Ramos A (2014) Estimación del diámetro, altura y volumen a partir del diámetro del tocón para Quercus laurina, en Ixtlán, Oaxaca, México. Madera y Bosques 20: 59-70.

Pretzsch H, Biber P, Uhl E, Dahlhausen J, Rötzer T, Caldentey J, et al. (2015) Crown size and growing space requirement of common tree species in urban centres, parks, and forests. Urban Forestry \& Urban Greening 14: 466-479.

Ramírez-Martínez A, Santiago-García W, Quiñonez-Barraza G, Ruiz-Aquino F, Martínez-Antúnez P (2016) Modelos de volumen fustal para Pinus ayacahuite Ehren. Revista Mexicana de Agroecosistemas 3: 61-74.

Romahn de la V CF, Ramírez-Maldonado H (2010) Dendrometría. Universidad Autónoma Chapingo. Texcoco, Estado de México, México. 354p.

Schumacher FX, Hall FD (1933) Logarithmic expression of timber-tree volume. Journal of Agricultural Research 47: 719-734.

Sosa-Escudero W (2005) Perspectivas y avances recientes en regresión por cuantiles. En: Marchionni M, Ahumada H, Jorrat JM, Navarro AM, Sosa-Escudero W (ed.) Progresos en econometría. Temas Grupo Editorial. Buenos Aires, Argentina. pp: 101-133.

Wardasanti CE (2011) Persamaan Penduga Volume Pohon Pinus (Pinus merkusii Jungh et de Vriese) di Hutan Pendidikan Gunung Walat Kabupaten Sukabumi, Jawa Barat. http://repository.ipb.ac.id/bitstream/handle/123456789/52859/E11cew.pdf?sequence=9\&isAllowed=y Fecha de consulta: 20 de abril de 2018. 
Weeraratne NC (2016) The effects of multicollinearity in ordinary least squares (ols) estimation. Department of Economics \& Statistics SUSL, BelihulOya, Sri Lanka. http://ijariie.com/AdminUploadPdf/The Effects_of_Multicollinearity_in_Ordinary_Least_Squares__OLS_Estimation_ijariie2996.pdf. Fecha de consulta: 13 de abril de 2018.

Zimmerman DW (1994) A note on the influence of outliers on parametric and nonparametric tests. The journal of General Psychology 121: 391-401. 